\title{
(TA) UGT 1A1 Promoter Polymorphism: A Crucial Factor in the Pathophysiology of Jaundice in G-6-PD Deficient Neonates
}

\author{
MICHAEL KAPLAN, PAUL RENBAUM, HENDRIK J. VREMAN, RONALD J. WONG, EPHRAT LEVY-LAHAD, \\ CATHY HAMMERMAN, AND DAVID K. STEVENSON
}

\begin{abstract}
Departments of Neonatology [M.K., C.H.] and Clinical Genetics [P.R., E.L.-L.], Shaare Zedek Medical Center, Jerusalem 91031, Israel; Faculty of Medicine of the Hebrew University [M.K., E.L.-L.], Jerusalem 91031, Israel; Faculty of Health Sciences [C.H.], Ben Gurion University of the Negev, Be'er Sheva 84105, Israel; Department of Pediatrics [H.J.V., R.J.W., D.K.S.], Stanford University School of Medicine, Stanford, California 94304
\end{abstract}

\begin{abstract}
Increased heme catabolism has been reported in glucose-6-phosphate dehydrogenase (G-6-PD)-normal neonates who were also homozygous for $(\mathrm{TA})_{7} /(\mathrm{TA})_{7}(\mathrm{UGT} 1 \mathrm{~A} 1 * 28)$ uridine diphosphoglucuronate-glucuronosyltransferase 1A1 (UGT) promoter polymorphism (Gilbert syndrome). As G-6-PD deficiency is associated with increased hemolysis, we hypothesized that in G-6-PDdeficient neonates who also have the $(\mathrm{TA})_{7} /(\mathrm{TA})_{7}$ UGT promoter genotype, steady-state hemolysis would be even further increased. Male G-6-PD-deficient neonates were sampled for plasma total bilirubin (PTB), blood carboxyhemoglobin corrected for inhaled carbon monoxide in ambient air (COHbc) (an index of heme catabolism), and UGT (TA) n promoter genotype determination and compared with previously published G-6-PD-normal neonates. Although $\mathrm{COHbc}$ values were higher in the G-6-PD-deficient than in the G-6PD-normal cohorts $(0.97 \pm 0.32 \%$ of total $\mathrm{Hb}(\mathrm{tHb})$ versus $0.76 \pm$ $0.19 \%$ of tHb, $p<0.001)$, PTB values were similar $(9.2 \pm 3.4 \mathrm{mg} / \mathrm{dL}$ versus $8.9 \pm 3.0 \mathrm{mg} / \mathrm{dL}$, respectively, $p=0.3$ ). Within the G-6-PDdeficient group, although $\mathrm{COHbc}$ values were alike between the three UGT promoter genotypes, PTB was higher in the $(\mathrm{TA})_{7} /(\mathrm{TA})_{7}$ homozygotes $(11.1 \pm 4.0 \mathrm{mg} / \mathrm{dL})$ compared with $(\mathrm{TA})_{6} /(\mathrm{TA})_{7}$ heterozygotes $(9.1 \pm 3.2 \mathrm{mg} / \mathrm{dL}, p=0.03)$ and wild-type $(\mathrm{TA})_{6} /(\mathrm{TA})_{6}$ homozygotes $(8.8 \pm 3.4 \mathrm{mg} / \mathrm{dL}, p=0.02)$. In the steady state, similar rates of hemolysis, but increased PTB in the G-6-PD-deficient, $(\mathrm{TA})_{7} /(\mathrm{TA})_{7}$ homozygotes, imply that $(\mathrm{TA})_{7} /(\mathrm{TA})_{7}$ homozygosity is central to increased PTB. (Pediatr Res 61: 727-731, 2007)
\end{abstract}

$\mathrm{H}^{2}$ omozygosity for the $(\mathrm{TA})_{7}$ promoter polymorphism (also known as UGT1A $1 * 28$ ) in the gene encoding the bilirubin-conjugating enzyme UGT is associated with Gilbert syndrome and typically results in diminished enzyme expression with resultant reduced bilirubin conjugation when compared with the wild-type $\left[(\mathrm{TA})_{6} /(\mathrm{TA})_{6}\right]$ or heterozygotes $\left[(\mathrm{TA})_{6} /(\mathrm{TA})_{7}\right](1,2)$. Each additional $(\mathrm{TA})$ sequence in the promoter has been shown to diminish promoter activity and hence gene expression (3). Although Gilbert syndrome is not associated with overt hemolysis (4-6), some studies have

Received September 27, 2006; accepted January 30, 2007.

Correspondence: Michael Kaplan, M.B., Ch.B., Department of Neonatology, Shaare Zedek Medical Center, PO Box 3235, Jerusalem 91031, Israel; e-mail: kaplan@cc. huji.ac.il

Supported at Shaare Zedek Medical Center by grants from the Golden Charitable Trust, London, UK, and the Mirsky Research Fund and at Stanford University by National Institutes of Health grant RR00070, the Hess Research Fund, the H.M. Lui Research Fund, and the Mary L. Johnson Research Fund.

DOI: $10.1203 /$ pdr.0b013e31805365c5 shown decreased erythrocyte survival, implying of increased heme catabolism, in association with the condition (7-14). Accordingly, in a recently described G-6-PD-normal newborn cohort, (TA) 7 homozygosity, when compared with (TA) 6 homozygosity or (TA $)_{6} /(\mathrm{TA})_{7}$ heterozygosity, was associated, as expected, with increased serum total bilirubin and diminished serum total conjugated bilirubin (the latter indicative of decreased conjugation). In addition, $\mathrm{COHbc}$, an index of heme catabolism, was elevated in the $(\mathrm{TA})_{7} /(\mathrm{TA})_{7}(15)$.

G-6-PD deficiency is a commonly occurring enzymatic condition associated with a three- to fivefold increased rate of neonatal hyperbilirubinemia when compared with G-6-PDnormal counterparts $(16,17)$. When the jaundice is extreme, bilirubin encephalopathy with potentially devastating, permanent neurologic sequelae (kernicterus) may ensue.

A fundamental concept of bilirubin kinetics is that the serum bilirubin concentration at any point in time reflects an equilibrium between bilirubin production and its elimination. In the past, there was some debate as to whether increased hemolysis or diminished conjugation was the primary factor in the pathophysiology of hyperbilirubinemia in G-6-PD deficient neonates $(18,19)$. Recent studies have clarified the situation and shown that, despite increased hemolysis associated with the condition $(17,20,21)$, the icterus appears to be due to a predilection for diminished bilirubin conjugation compared with G-6-PD-normal counterparts $(22,23)$. The diminished bilirubin conjugation is the result of UGT $(\mathrm{TA})_{7}$ promoter polymorphism (24). A similar key role of UGT promoter polymorphism has been demonstrated in adults (25). In Asian neonates, an interaction between G-6-PD deficiency and coding area mutations of the UGT1A1 gene predispose to hyperbilirubinemia (26). However, these studies did not include measurements of bilirubin production.

As baseline hemolysis is increased in G-6-PD-deficient neonates $(17,20,21)$, and in light of the increased hemolysis found in G-6-PD-normal neonates who were homozygous for the $(\mathrm{TA})_{7}$ promoter polymorphism, we hypothesized that in

\footnotetext{
Abbreviations: COHb, carboxyhemoglobin; COHbc, carboxyhemoglobin corrected for inhaled carbon monoxide in ambient air; G-6-PD, glucose-6phosphate dehydrogenase; PTB, plasma total bilirubin; tHb, total hemoglobin; UGT, uridine diphosphoglucuronate-glucuronosyltransferase 1A1
} 
G-6-PD-deficient newborns, heme catabolism would be increased to an even greater extent than in G-6-PD-normal controls of the identical UGT promoter genotype. Our objective in this study was to further elucidate, in steady state, the respective roles, individually and in combination, of heme catabolism and (TA) $)_{7}$ UGT promoter polymorphism in determining PTB concentrations in a G-6-PD-deficient male neonatal cohort. For this purpose, blood $\mathrm{COHbc}$ and PTB were analyzed in relation to the three UGT promoter genotypes: $\left[(\mathrm{TA})_{6} /(\mathrm{TA})_{6}\right.$ homozygotes, $(\mathrm{TA})_{6} /(\mathrm{TA})_{7}$ heterozygotes, and $(\mathrm{TA})_{7} /(\mathrm{TA})_{7}$ homozygotes].

\section{METHODS}

The current G-6-PD-deficient neonatal cohort was enrolled in two time periods. The first, sampled concurrently with the previously published G-6PD-normal cohort (15) between 1997 and 1999, yielded 131 G-6-PD-normal neonates, but only 52 G-6-PD-deficient newborns. The G-6-PD-deficient cohort was expanded between 2002 and 2003 to obtain an overall sample size similar to that of the G-6-PD-normal cohort. We predicted that the UGT promoter subgroups among the G-6-PD-deficient neonates would be of similar size to the UGT promoter subgroups in the G-6-PD-normal cohort and that similar subgroup sizes would facilitate comparisons between subgroups of comparable numbers.

As the methodology was identical to that of the first study, salient features only of the protocol are repeated here. The study protocol was approved by the Institutional Review Board of the Shaare Zedek Medical Center. The patient cohort consisted of healthy, consecutively born male neonates delivered at $\geq 37$ wk gestational age to Sephardic Jewish mothers at the Shaare Zedek Medical Center. All babies were tested for G-6-PD Mediterranean, the mutation known to occur in this population subgroup $(24,27)$, and only those with this mutation were included in the current analysis. Males only were studied so as to avoid encountering the G-6-PD heterozygous state that may be present in females, which cannot be diagnosed by regular biochemical tests and is more difficult to perform even by molecular methods (19). Also excluded were infants with other conditions that may have affected bilirubin metabolism, including direct Coombs'-positive hemolytic disease, sepsis, maternal diabetes, cephalhematoma or extensive bruising, and Down syndrome. All babies received routine clinical management during their nursery stay.

Blood was sampled for the study on the third day of life, concomitant with routine predischarge metabolic screening, to avoid a blood-taking procedure solely for the purpose of the study. Whole blood for carboxyhemogloboin $(\mathrm{COHb})$ and total $\mathrm{Hb}(\mathrm{tHb})$ determination $(150 \mu \mathrm{L})$ was collected into custom-made capillary tubes containing heparin and saponin, as previously described $(28,29)$, and stored at $-18^{\circ} \mathrm{C}$. For ease of shipping, the samples were removed from the freezer and transported on ice to Stanford University. Simultaneously with the blood $\mathrm{COHb}$ collection, a sample of room air from the nursery in which the baby was being cared for was collected and stored in a special stainless steel container until determination of its $\mathrm{CO}$ concentration (Bistable Gas Sampler, Chemical Projects Ltd., Toronto, ON, Canada). Blood for PTB was collected into microcapillary tubes, and for DNA extraction, for subsequent UGT promoter analysis, and G-6-PD Mediterranean determination, into ethylenediamine tetraacetic acid-containing tubes.

Laboratory methods. $\mathrm{COHb}$ was determined at Stanford University by a gas chromatographic method and its concentrations expressed as a percentage of $\mathrm{tHb}$, which was quantitated by the cyanmethemoglobin method, as previously described $(28,29)$. Using this method, the within-day and between-day coefficients of variation for reference blood samples are $3 \%$ and $8 \%$, respectively (30). The $\mathrm{CO}$ concentration of the room air specimens was determined at the Shaare Zedek Medical Center using a sensitive electrochemical CO analyzer supplied by Stanford University (31). Room air CO concentrations, representing inhaled $\mathrm{CO}$, which ranged from 0.15 to 1.33 parts per million (mean, 0.46) were used to correct measured $\mathrm{COHb}$ for the effect of inspired $\mathrm{CO}$ by a previously derived formula $(\mathrm{COHbc}=$ measured $\mathrm{COHb}-0.17(\mu \mathrm{L}$ $\mathrm{CO} / \mathrm{L}$ room air) (32). Results of $\mathrm{COHb}$ analysis by gas chromatography have shown that the samples, studied for quality control, are stable for up to at least $5 \mathrm{mo}$ at $4^{\circ} \mathrm{C}$, and change very little when stored at ambient temperature (29). The tubes are $>90 \%$ filled and are sealed hermetically. The samples are therefore stable under most conditions of storage and transport and are suited for analysis after long-distance transportation.

PTB was measured routinely in the hospital's clinical biochemistry laboratory by a direct spectrophotometric method using spun capillary tube samples (Wako Bilirubin Tester, Wako Pure Chemical Industries, Ltd., Osaka, Japan).

DNA was prepared from peripheral leukocytes by a salt extraction method (33). The presence or absence of nt 563, the nucleotide mutated in G-6-PD Mediterranean, was determined at the Scripps Research Institute, La Jolla, CA (courtesy Ernest Beutler, M.D.) by polymerase chain reaction followed by allele-specific oligonucleotide hybridization, as published elsewhere (24). UGT promoter genotype (UGT1A1*28, refSNP ID rs8175347) was determined at Shaare Zedek Medical Center by PCR-mediated, site-directed mutagenesis, as previously described (24)

Data analysis. UGT genotypes were assigned according to the gene promoter TATAA box sequence: neonates bearing the sequence $[\mathrm{TA}]_{6}$ TAA in the promoter of both alleles were classified as normal homozygotes, those with the sequence $[\mathrm{TA}]_{7}$ TAA in both alleles as variant homozygotes (the UGT promoter genotype associated with Gilbert syndrome), and those with one of each allele as heterozygotes $(1,2)$.

The continuous variables PTB and COHbc across the three UGT promoter genotype groups were compared by analysis of variance (ANOVA) as an initial statistical test. In the event of significance $(p<0.05)$, the ANOVA was followed by the Student-Newman-Keuls test or Dunn's test, as appropriate, to determine which variables contributed to the significance. Further statistical analysis was performed using a $t$ test. Parametric data are presented as mean $\pm \mathrm{SD}$. Data from the previously published G-6-PDnormal cohort are presented for reference, with permission from Hepatology (15), and compared with the current cohort.

Hyperbilirubinemia was defined as any PTB concentration $>95$ th percentile for age in hours according to the hour of life-specific bilirubin nomogram (34).

\section{RESULTS}

This G-6-PD-deficient male cohort comprised 131 neonates [mean $( \pm \mathrm{SD})$ birth weight $3219 \pm 400 \mathrm{~g}$, mean gestational age $39.3 \pm 1.4 \mathrm{wk}$. Sixteen $(12 \%)$ were born by cesarean section. One hundred two (82\%) received breast milk either exclusively or in conjunction with formula. Allele frequency for the (TA) $)_{7}$ UGT promoter was 0.37. Genotype distribution is shown in Table 1. Average age at sampling was $58 \pm 12 \mathrm{~h}$. Although the mean $\mathrm{COHbc}$ value for the cohort was higher in these G-6-PD-deficient neonates than in the previously published G-6-PD-normal neonates (0.97 \pm $0.32 \%$ of $\mathrm{tHb}$ versus. $0.76 \pm 0.19 \%$ of $\mathrm{tHb}, p<0.001)$, mean PTB concentration for the cohort was not significantly higher $(9.2 \pm 3.4 \mathrm{mg} / \mathrm{dL}$, compared with $8.9+3.0 \mathrm{mg} / \mathrm{dL}$ for the G-6-PD-normal newborns, $p=0.3$ ) (Fig. 1, left bar graphs).

In the G-6-PD-normal group, as previously published, $\mathrm{COHbc}$ values and PTB concentrations were higher in the

Table 1. PTB and COHbc values in G-6-PD-deficient neonates stratified for UGT promoter polymorphism

UGT promoter genotype

\begin{tabular}{|c|c|c|c|c|}
\hline & \multicolumn{2}{|c|}{ UGT promoter genotype } & \multirow[b]{2}{*}{$(\mathrm{TA})_{7} /(\mathrm{TA})_{7}$} & \multirow[b]{2}{*}{ Significance } \\
\hline & $(\mathrm{TA})_{6} /(\mathrm{TA})_{6}$ & $(\mathrm{TA})_{6} /(\mathrm{TA})_{7}$ & & \\
\hline No. of neonates (131) & $53(40.5 \%)$ & $60(45.8 \%)$ & $18(13.7 \%)$ & \\
\hline PTB $(m g / d L)$ & $8.8 \pm 3.4$ & $9.1 \pm 3.2$ & $11.1 \pm 4.0^{*}$ & $\begin{aligned} p & =0.02\left[(\mathrm{TA})_{7} /(\mathrm{TA})_{7} v s(\mathrm{TA})_{6} /(\mathrm{TA})_{6}\right] \\
p & =0.03\left[(\mathrm{TA})_{7} /(\mathrm{TA})_{7} v s(\mathrm{TA})_{6} /(\mathrm{TA})_{7}\right]\end{aligned}$ \\
\hline $\mathrm{COHbc}(\%$ of $\mathrm{tHb})$ & $0.95 \pm 0.31$ & $0.98 \pm 0.33$ & $1.01 \pm 0.31$ & NS \\
\hline
\end{tabular}




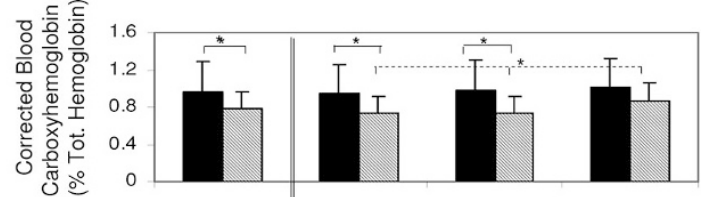

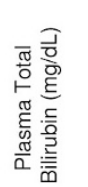

UGT Promoter Genotype:

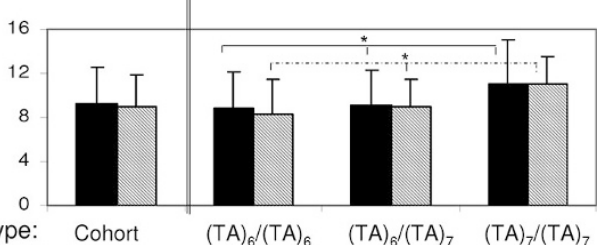

Figure 1. COHbc values (top), and $\mathrm{PTB}$ concentrations (bottom) for neonates of the G-6-PD-deficient $(n=131)$ (solid columns) and -normal ( $n=131$ ) (hatched columns) cohorts, respectively, and three UGT promoter genotypes. Despite higher $\mathrm{COHbc}$, indicating increased hemolysis, for the G-6-PD-deficient neonates of the cohort $(p<0.001)$ as well as the (TA) 6 / $(\mathrm{TA})_{6}(p<0.001)$ and $(\mathrm{TA})_{6} /(\mathrm{TA})_{7}(p<0.001)$ UGT promoter genotype subgroups, PTB concentrations were similar between the G-6-PD-deficient and G-6-PD-normal neonates. Although the COHbc values in the G-6-PDdeficient neonates were similar in all three UGT promoter genotypes, PTB was higher in the $(\mathrm{TA})_{7} /(\mathrm{TA})_{7}$ subgroup compared with $(\mathrm{TA})_{6} /(\mathrm{TA})_{6}$ and $(\mathrm{TA})_{6} /(\mathrm{TA})_{7}$ subgroups $(p=0.02$ and 0.03 , respectively). This finding indicates a crucial role for $(\mathrm{TA})_{7} /(\mathrm{TA})_{7}$ UGT promoter polymorphism in determining PTB concentrations in G-6-PD-deficient neonates. (TA) 6 / (TA) 6 , homozygosity for the wild-type UGT promoter; $(\mathrm{TA})_{6} /(\mathrm{TA})_{7}$, heterozygote UGT promoter; (TA $)_{7} /(\mathrm{TA})_{7}$, homozygosity for the variant UGT promoter.

$(\mathrm{TA})_{7} /(\mathrm{TA})_{7}$ homozygotes only, compared with the wildtype $(\mathrm{TA})_{6} /(\mathrm{TA})_{6}$ homozygotes or $(\mathrm{TA})_{6} /(\mathrm{TA})_{7}$ heterozygotes (15). In contrast, in the G-6-PD-deficient group, although there was a tendency for $\mathrm{COHbc}$ values to increase with each additional (TA) sequence, the generally higher $\mathrm{COHbc}$ values were not significantly different between the three UGT (TA) promoter genotype subgroups (Fig. 1 and Table 1). In contrast, PTB was higher in the $(\mathrm{TA})_{7} /(\mathrm{TA})_{7}$ homozygotes $(11.1 \pm 4.0 \mathrm{mg} / \mathrm{dL})$ compared with both $(\mathrm{TA})_{6} /(\mathrm{TA})_{7}$ heterozygotes $(9.1 \pm 3.2 \mathrm{mg} / \mathrm{dL}$, $p=0.03)$ and $(\mathrm{TA})_{6} /(\mathrm{TA})_{6}$ homozygotes $(8.8 \pm 3.4 \mathrm{mg} / \mathrm{dL}$, $p=0.02$ ) (Fig. 1). Correspondingly, 0 of $53(0 \%)$ of the $(\mathrm{TA})_{6} /(\mathrm{TA})_{6}$ homozygotes, four of $60(6.6 \%)$ of the $(\mathrm{TA})_{6} /(\mathrm{TA})_{7}$ heterozygotes, and three of $18(16.6 \%)$ of the $(\mathrm{TA})_{7} /(\mathrm{TA})_{7}$ homozygotes were hyperbilirubinemic at the time of sampling.

Between groups, COHbc values of the G-6-PD-deficient neonates were significantly higher in the (TA $)_{6} /(\mathrm{TA})_{6}(p<$ $0.001)$ and $(\mathrm{TA})_{6} /(\mathrm{TA})_{7}(p<0.001)$ promoter genotypes than in the equivalent, previously published G-6-PDnormal counterparts, with a trend toward increased values in the G-6-PD-deficient (TA $)_{7} /(\mathrm{TA})_{7}$ homozygotes as well $(p=0.09)$ (Fig. 1). However, despite this increased hemolysis, PTB values were virtually identical between G-6-PDdeficient and -normal neonates, within the various UGT promoter subgroups. Within the groups, PTB values were increased only in the (TA $)_{7} /(\mathrm{TA})_{7}$ homozygotes compared with equivalent $(\mathrm{TA})_{6} /(\mathrm{TA})_{6}$ and $(\mathrm{TA})_{6} /(\mathrm{TA})_{7}$ subgroups. In the G-6-PD-deficient group, in light of the similarity between $\mathrm{COHbc}$ values between the three UGT promoter subgroups, by inference, the higher PTB concentrations in the G-6-PD-deficient (TA $)_{7} /(\mathrm{TA})_{7}$ homozygotes must have been mediated by decreased bilirubin conjugation associated with this promoter genotype.

\section{DISCUSSION}

As expected from previous studies $(17,20,21)$, COHbc values were significantly higher in the G-6-PD-deficient cohort than in the controls. However, despite the increased heme catabolism, PTB concentrations at the time of sampling were similar between the two groups. This finding demonstrates a relatively minor role of increased heme catabolism in the pathophysiology of the increased PTB concentrations in G-6PD-deficient neonates.

On subgroup analysis, within the G-6-PD-deficient group, despite a tendency to higher $\mathrm{COHbc}$ values with the addition of (TA) sequences, heme catabolism was in fact similar between the three UGT 1A1 promoter genotypes. However, in contrast, PTB was significantly higher in the $(\mathrm{TA})_{7} /(\mathrm{TA})_{7}$ homozygotes. As the PTB at any point in time is dependent on the equilibrium between bilirubin production and elimination, by inference, homozygosity for the (TA $)_{7}$ promoter subtype must have been central to the increased PTB concentrations found in the $(\mathrm{TA})_{7} /(\mathrm{TA})_{7}$ homozygotes. This constellation differs from the G-6-PD-normal group, in whom both increased heme catabolism and (TA $)_{7} /(\mathrm{TA})_{7}$ homozygosity may have played complementary roles in the pathogenesis of the jaundice. In a previous study, we demonstrated that the presence of the variant UGT promoter was crucial to the development of hyperbilirubinemia in G-6-PD-deficient neonates (24). Our current findings demonstrate the importance of decreased bilirubin conjugation, relative to increased heme catabolism, in the development of steady state, and moderately elevated PTB levels in G-6-PD-deficient neonates as well.

In parallel with what we had found in the G-6-PD-normal neonates (15), we hypothesized that in the G-6-PD-deficient neonates $\mathrm{COHbc}$ values would be even further increased in the UGT (TA $)_{7} /(\mathrm{TA})_{7}$ homozygote subgroup than the wildtype and heterozygotes and also greater than the equivalent promoter genotype of the G-6-PD-normal group. Surprisingly, therefore, the generally higher rate of heme catabolism was not significantly higher, as expected, in the $(\mathrm{TA})_{7} /(\mathrm{TA})_{7}$ homozygotes. Between groups, however, $\mathrm{COHbc}$ values were significantly higher in both the G-6-PD-deficient (TA) ${ }_{6} /(\mathrm{TA})_{6}$ homozygotes and $(\mathrm{TA})_{6} /(\mathrm{TA})_{7}$ heterozygotes compared with the G-6-PD-normal counterparts, with a trend between G-6PD-deficient and G-6-PD-normal (TA) ${ }_{7} /(\mathrm{TA})_{7}$ homozygotes. The lack of significance in the latter analysis was most likely due to increased baseline hemolysis in the G-6-PD-normal $(\mathrm{TA})_{7} /(\mathrm{TA})_{7}$ neonates, reflecting shorter red cell life span associated with the Gilbert syndrome polymorphism (15). The reason for the dissimilarity in the pattern of $\mathrm{COHbc}$ values between the three UGT promoter genotypes in the G-6-PDdeficient and -normal groups is not clear. The subtle, yet significant, increase in hemolysis observed in G-6-PD-normal infants with the (TA) $)_{7} /(\mathrm{TA})_{7}$ genotype (15) may have been masked by the high basal hemolytic rate associated with G-6-PD deficiency. We speculate that the processes contributing 
to increased heme catabolism in the G-6-PD-normal (TA) ${ }_{7} /$ (TA $)_{7}$ homozygotes were overwhelmed by the mechanisms leading to even higher baseline hemolysis in the G-6-PD deficiency state. Because there was similar hemolysis in the $(\mathrm{TA})_{7} /(\mathrm{TA})_{7}$ subgroups of both the G-6-PD-deficient and -normal neonates, we cannot assess whether $(\mathrm{TA})_{7} /(\mathrm{TA})_{7}$ homozygosity independently, in the absence of increased hemolysis, would have resulted in increased PTB concentrations.

In this study, we examined only the (TA) $)_{n}$ allele of the UGT1A1 gene promoter. This UGT1A1*28 mutation is the most common mutation associated with Gilbert syndrome in Caucasian populations and has an established association with neonatal hyperbilirubinemia. Further information on this polymorphism can be found at http://som.flinders.edu.au/FUSA/ ClinPharm/UGT/1A1alleles.html. The only other UGT mutation associated with Gilbert syndrome and with neonatal hyperbilirubinemia is the G71R mutation (35). However, this mutation is rare in Caucasians and is encountered primarily in Asian populations. Other UGT mutations associated with Gilbert syndrome were not studied as they have not been demonstrated to be associated with neonatal hyperbilirubinemia (36). The genotype distribution of the UGT promoter (TA) n sequence cannot be expected to be in HardyWeinberg equilibrium. Although we enrolled only males whose Sephardic Jewish mother's families had immigrated to Israel from geographic areas in the Middle East, the communities from which they came had been widely scattered for two millennia. During the centuries of dispersion, there was most likely some intermarriage with the local populations. Since these groups immigrated to Israel starting in the early 1950s, there has been a growing trend toward intermarriage between population subgroups. The population we studied, therefore, cannot be considered a to represent a homogeneous group.

A unique aspect of this study is that the design enabled us to simultaneously assess heme catabolism as well as the effect of the UGT promoter polymorphism in the same individuals. Our data show that, although steady-state PTB concentrations were similar at the time of sampling, the processes contributing to PTB, especially bilirubin production, were different in G-6-PD-deficient neonates than in those with normal enzyme activity. It is important to realize that, although PTB concentrations do not usually reach exceptionally high levels, in the G-6-PD-deficient state, heme catabolism functions at a higher rate than normal, and diminished bilirubin conjugation plays a major role. These forces may exacerbate the lack of equilibrium between bilirubin production and elimination. Should these newborns be exposed to additional oxidative stressors, or have further compromise of the bilirubin conjugating system, the additional resultant bilirubin load might exceed the body's ability to eliminate that substance, with the potential of severe hyperbilirubinemia. In the future, implementation of a genetic screening test with rapid turnabout time might be effective in complementing G-6-PD screening, thereby further pinpointing those neonates at high risk of developing hyperbilirubinemia.
Acknowledgments. The authors thank Ernest Beutler, M.D., for G-6-PD genotyping.

\section{REFERENCES}

1. Bosma PJ, Chowdhury JR, Bakker C, Gantla S, de Boer A, Oostra BA, Lindhout D, Tytgat GN, Jansen PL, Oude Elferink RP, Chowdhury NR 1995 The gene basis of the reduced expression of bilirubin UDP-glucuronosyltransferase 1 in Gilbert's syndrome. N Engl J Med 333:1171-1175

2. Monaghan G, Ryan M, Seddon R, Hume R, Burchell B 1996 Genetic variation in bilirubin UDP-glucuronosyltransferase gene promoter and Gilbert's syndrome. Lancet 347:578-581

3. Beutler E, Gelbart T, Demina A 1998 Racial variability in the UDPglucuronosyltransferase 1 (UGT1A1) promoter: a balanced polymorphism for regulation of bilirubin metabolism? Proc Natl Acad Sci U S A 95:8170-8174

4. Roy-Chowdhury J, Jansen PL 1996 Bilirubin metabolism and its disorders. In: Zakim D, Boyer TD (eds) A Text Book of Liver Disease. WB Saunders, Philadelphia, pp 323-361.

5. Burchell B, Hume R 1999 Molecular genetic basis of Gilbert's syndrome. J Gastroenterol Hepatol 14:960-966

6. Clarke DJ, Moghrabi N, Monaghan G, Cassidy A, Boxer M, Hume R Burchell 1997 Genetic defects of the UDP-glucuronosyltransferase-1 (UGT1) gene that cause familial non-haemolytic unconjugated hyperbilirubinaemias. Clin Chim Acta 266:63-74.

7. Foulk WT, Butt HR, Owen CR, Whitcomb FF, Mason HL 1959 Constitutional hepatic dysfunction (Gilbert's Disease): Its natural history and related syndromes. Medicine 38:25-46

8. Arias IM 1962 Chronic unconjugated hyperbilirubinemia without overt signs of hemolysis in adolescents and adults. J Clin Invest 41:2233-2245

9. Pitcher CS, Williams R 1963 Reduced red cell survival in jaundice and its relation to abnormal glutathione metabolism. Clin Sci 24:239-252

10. Powell LW, Billing BH, Williams HS 1967 An assessment of red cell survival in idiopathic unconjugated hyperbilirubinaemia (Gilbert's syndrome) by the use of radioactive isopropylfluorophosphate and chromium. Australas Ann Med 16:221225

11. Powell LW, Hemingway E, Billing BH, Sherlock S 1967 Idiopathic unconjugated hyperbilirubinemia (Gilbert's syndrome). A study of 42 families. N Engl J Med 277:1108-1112

12. Berk PD, Blaschke TF 1972 Detection of Gilbert's syndrome in patients with hemolysis. A method using radioactive chromium. Ann Intern Med 77:527-531

13. Bensinger TA, Maisels MJ, Carlson DE, Conrad ME 1973 Effect of low caloric diet on endogenous carbon monoxide production: normal adults and Gilbert's syndrome. Proc Soc Exp Biol Med 144:417-419

14. Okolicsanyi L, Ghidini O, Orlando R, Cortelazzo S, Benedetti G, Naccarato R, Manitto P 1978 An evaluation of bilirubin kinetics with respect to the diagnosis of Gilbert's syndrome. Clin Sci Mol Med 54:539-547

15. Kaplan M, Hammerman C, Rubaltelli FF, Vilei MT, Levy-Lahad E, Renbaum P, Vreman HJ, Stevenson DK, Muraca M 2002 Hemolysis and bilirubin conjugation in association with UDP-glucuronosyltransferase 1A1 promoter polymorphism. Hepatology 35:905-911

16. Kaplan M, Algur N, Hammerman C 2001 Onset of jaundice in glucose-6-phosphate dehydrogenase-deficient neonates. Pediatrics 108:956-959

17. Kaplan M, Herschel M, Hammerman C, Hoyer JD, Stevenson DK 2004 Hyperbilirubinemia among African American, glucose-6-phosphate dehydrogenase-deficient neonates. Pediatrics 114:e213-e219

18. Valaes T 1994 Severe neonatal jaundice associated with glucose-6-phosphate dehydrogenase deficiency: pathogenesis and global epidemiology. Acta Paediatr Suppl 394:58-76

19. Beutler E 1994 G6PD deficiency. Blood 84:3613-3636

20. Kaplan M, Vreman HJ, Hammerman C, Leiter C, Abramov A, Stevenson DK 1996 Contribution of haemolysis to jaundice in Sephardic Jewish glucose-6-phosphate dehydrogenase deficient neonates. Br J Haematol 93:822-827

21. Kaplan M, Hammerman C, Renbaum P, Levy-Lahad E, Vreman HJ, Stevenson DK 2001 Differing pathogenesis of perinatal bilirubinemia in glucose-6-phosphate dehydrogenase-deficient versus-normal neonates. Pediatr Res 50:532-537

22. Kaplan M, Rubaltelli FF, Hammerman C, Vilei MT, Leiter C, Abramov A, Muraca M 1996 Conjugated bilirubin in neonates with glucose-6-phosphate dehydrogenase deficiency. J Pediatr 128:695-697

23. Kaplan M, Muraca M, Hammerman C, Vilei MT, Leiter C, Rudensky B, Rubaltelli FF 1998 Bilirubin conjugation, reflected by conjugated bilirubin fractions, in glucose-6-phosphate dehydrogenase-deficient neonates: a determining factor in the pathogenesis of hyperbilirubinemia. Pediatrics 102:E37

24. Kaplan M, Renbaum P, Levy-Lahad E, Hammerman C, Lahad A, Beutler E 1997 Gilbert's Syndrome and glucose-6-phosphate dehydrogenase deficiency: A dose dependent genetic interaction crucial to neonatal hyperbilirubinemia. Proc Natl Acad Sci U S A 94:12128-12132

25. Sampietro M, Lupica L, Perrero L, Comino A, Martinez di Montemuros F, Cappellini MD, Fiorelli G 1997 The expression of uridine diphosphate glucuronosyltransferase gene is a major determinant of bilirubin level in heterozygous betathalassaemia and in glucose-6-phosphate dehydrogenase deficiency. Br J Haematol 99:437-439

26. Huang CS, Chang PF, Huang MJ, Chen ES, Chen WC 2002 Glucose-6-phosphate dehydrogenase deficiency, the UDP-glucuronosyl transferase 1A1 gene, and neonatal hyperbilirubinemia. Gastroenterology 123:127-133 
27. Oppenheim A, Jury CL, Rund D, Vulliamy TJ, Luzzatto L 1993 G6PD Mediterranean accounts for the high prevalence of G6PD deficiency in Kurdish Jews. Hum Genet 91:293-294

28. Vreman HJ, Kwong LK, Stevenson DK 1984 Carbon monoxide in blood: an improved microliter blood-sample collection system, with rapid analysis by gas chromatography. Clin Chem 30:1382-1385

29. Vreman HJ, Stevenson DK, Zwart A 1987 Analysis for carboxyhemoglobin by gas chromatography and multicomponent spectrophotometry compared. Clin Chem 33:694-697

30. Widness JA, Lowe LS, Stevenson DK, Vreman HJ, Weiner CP, Hayde M, Pollak A 1994 Direct relationship of fetal carboxyhemoglobin with hemolysis in alloimmunized pregnancies. Pediatr Res 35:713-719

31. Vreman HJ, Stevenson DK, Oh W, Fanaroff AA, Wright LL, Lemons JA, Wright E, Shankaran S, Tyson JE, Korones SB, et al 1994 Semiportable electrochemical instrument for determining carbon monoxide in breath. Clin Chem 40:1927-1933
32. Ostrander CR, Cohen RS, Hopper AO, Cowan BE, Stevenson DK 1982 Paired determinations of blood carboxyhemoglobin concentrations and carbon monoxide excretion rate in term and preterm infants. J Lab Clin Med 100 $745-755$

33. Miller SA, Dykes DD, Polesky HF 1988 A simple salting out procedure for extracting DNA from human nucleated cells. Nucleic Acids Res 16:1215

34. Bhutani VK, Johnson L, Sivieri EM 1999 Predictive ability of a predischarge hour-specific serum bilirubin for subsequent significant hyperbilirubinemia in healthy term and near-term newborns. Pediatrics 103:6-14

35. Maruo Y, Nishizawa K, Sato H, Doida Y, Shimada M 1999 Association of neonatal hyperbilirubinemia with bilirubin UDP-glucuronosyltransferase polymorphism. Pediatrics 103:1224-1227

36. Costa E 2006 Hematologically important mutations: bilirubin UDP-glucuronosyltransferase gene mutations in Gilbert and Crigler-Najjar syndromes. Blood Cells Mol Dis 36:77-80 